\title{
Network Pharmacology-Based Analysis on the Mechanism of Drug Pair of Astragalus Membranaceus and Acorus Tatarinowii in the Treatment of Alzheimer's Disease
}

\author{
Xiuting HUANG*, Xiujin ZHANG*, Xiangning LI, Haozhen WANG, Chen LU, Ziyin \\ LU and Xiuli LU ${ }^{1}$ \\ Department of Biochemistry and Cell Biology, School of Life Science, Liaoning \\ University, China
}

\begin{abstract}
Alzheimer's disease (AD) is a degenerative disease of central nervous system, which seriously threatens the life and health of the elderly people. It has been for long time that Traditional Chinese medicine (TCM) treatment for AD is effective. This study analyzed the potential target and molecular mechanism of the most often used drug pair of Astragalus membranaceus and Acorus tatarinowii to treat AD by network pharmacological method. Firstly, the method was performed to screen and sort out the active ingredients with good ADME properties and drug targets of Astragalus membranaceus and Acorus tatarinowii. Then, we searched for the disease targets related to $\mathrm{AD}$, followed by the construction of the "active ingredients-target-disease" network. We implemented GO enrichment analysis and KEGG pathway enrichment analysis of related overlapped target proteins, and then constructed the "target-pathway" network diagram. Finally, the above overlapped target proteins are mapped to build a PPI high-position protein interoperability network, and we conducted the network topology analysis to screen out the core targets of Astragalus membranaceus-Acorus tatarinowii drug pair in the treatment of AD. According to network pharmacology, this research predicted the potential targets of the drug pair of Astragalus membranaceus and Acorus tatarinowii in the treatment of $\mathrm{AD}$, and explored that Astragalus membranaceus-Acorus tatarinowii drug pair in the treatment of $\mathrm{AD}$ was the overall systematic regulating action of "multiple-ingredients, multiple-target and multiple-pathway". It affords the reference for understanding the pathogenesis of $\mathrm{AD}$ and exploring new therapeutic methods and drug development in the future.
\end{abstract}

Keywords. Alzheimer's disease, the network pharmacology, Astragalus membranaceus, Acorus tatarinowii, mechanism

\section{Introduction}

Alzheimer's disease (AD) is the main types senile dementia, accounting for about $60 \%$ of senile dementia [1]. Its clinical manifestations including progressive memory decline, cognitive dysfunction, other neurological problems and behavioral disorders [2].

${ }^{1}$ Corresponding Author, Xiuli LU, Department of Biochemistry and Cell Biology, School of Life Science, Liaoning University, China; Email: luxiuli@lnu.edu.cn.

* Co-First authors 
Studies have found that age is the main factor in the onset of AD in patients with AD. Among people aged 50-80 years, the prevalence of AD will double for every 5-year increase in age [3]. According to the epidemiological survey, with the rising trend of population aging in China, the incidence of AD will increase significantly. It expected that by 2030, there will be more than $10 \times 106$ cases of AD in China [4].

The pathological features of $\mathrm{AD}$ are Tau protein deposition [5] and intracellular neurofibrillary tangles [6]. At present, there are several theories about the pathogenesis of AD: cholinergic deficiency [7], hyperphosphorylation of Tau [8], oxidative stress and excessive deposition of $A \beta$ [9]. These theories provide an overview of the pathogenesis of $\mathrm{AD}$ from different aspects, but the etiology of the disease is extremely complex and its pathogenesis remains unclear.

The modern medical treatment of AD is mainly based on drug, supplemented by cognitive training. Medicine therapy mainly includes improving brain microcirculation, regulating nerve cell metabolism, reducing oxidative stress, repairing nerve fibers and so on. Common drugs for the treatment of AD include Donepezil [10], Sodium oligomannate [11], VitaminC [12] and Memantine [13]. Although modern western medicine can improve some symptoms of $\mathrm{AD}$ patients, it cannot fundamentally treat AD [14]. At present, some studies showed that Chinese herbal medicine can effectively treat $\mathrm{AD}$, such as Ginseng therapy, which protects neurons and improves cognitive function [15]. Salvia miltiorrhiza Bunge reduces Tau hyperphosphorylation and prevents the formation and decomposition of $A \beta$ fiber [16].

Astragalus membranaceus has the efficacy of improving body immunity, antiaging and anti-oxidation in the modern pharmacological study. Studies have found that Astragalus polysaccharide can alleviate the process of $\mathrm{AD}$ by downregulating the expression levels of APP, A $\beta$, PTAu, GSK3 $\beta$ and BACE1 in the hippocampus of AD model rats and up-regulating the protein expression level of PP2A, and can also significantly improve the positioning and navigate ability and learning and memory ability of rats [17]. The extracts, volatile oil and asarone ether of Acorus tatarinosus have sedative, anti-convulsant, anti-depression, improving learning and memory and anti-brain damage effects. Tian et al. [18] studied the mechanism of different effective parts of Acorus tatarinowii, improving the cognitive function of $\mathrm{AD}$ model mice, and found that the cognitive function of model mice was improved by gavage of different effective parts of Acorus tatarinowii, and the water extract and volatile oil gavage group improved the cognitive function of mice most significantly. Song [19] et al. conducted data mining on TCM prescriptions for the treatment of AD in the past 30 years. According to the research, among the 241 first prescriptions based on the statistics of "ancient and modern medical case cloud platform V1.4.1", the top five are Acorus tatarinowii, Astragalus membranaceus, Rehmannia glutinosa, Salvia miltiorrhiza, and Poria; The most common drug pairs are "Acorus tatarinowiiPolygala", "Acorus tatarinowii-Astragalus membranaceus" and "Acorus tatarinowiiSalvia miltiorrhiza". Based on the analysis of the law of TCM for 241 prescriptions, the confidence and correlation of the three-drug pairs are still in the top three.

Network pharmacology analyzes the multiple-level and multiple-angle biological network relationship among "medicine-gene-target-disease" and the relationship between different ingredients of TCM. It also studies the biological pathway signals of diseases, and establishes the overall relationship between active ingredients and targets and diseases. The integrity, systematization and emphasis on the interaction between drugs of the network pharmacological analysis are consistent with the characteristics of 
TCM, so as it can confirm potential drug targets and clarify the mechanism of drug action [20].

This study purposed to analyze the potential targets and molecular mechanisms of action of Astragalus membranaceus-Acorus tatarinowii drug pair in the treatment of $\mathrm{AD}$ by the network pharmacological method. It supplies a theoretical foundation and research strategies for future research on the pathogenesis of $\mathrm{AD}$, as well as the research on new therapeutic methods and drug development.

\section{Materials and Methods}

\subsection{Screening of Active Ingredients of Drug Pair}

This paper used the Traditional Chinese Medicine Systems Pharmacology Database (TCMSP) (http://tcmspw.com/tcmsp.php) to collect the active ingredients of Astragalus membranaceus and Acorus tatarinowii. Taking "Astragalus membranaceus" and "Acorus tatarinowii" as the search key words, Drug-likeness (DL $\geq 0.18$ ), Blood-brain barrier ( $\mathrm{BBB} \geq-0.3)$, and Oral Bioavailability $(\mathrm{OB} \geq 30 \%)$ as the search conditions, then acquired the active ingredients of Astragalus membranaceus and Acorus tatarinowii for treating $\mathrm{AD}$.

\subsection{Collection of Disease Targets Related to Alzheimer's Disease}

To explore the relevant disease targets of $\mathrm{AD}$, we used the DisGeNET (http://www.Disgenet.org/) database to obtain the target genes related to AD by using the medical keyword “Alzheimer's Disease". Subsequently, sort out the collected relevant disease targets, and then obtain the relevant known human targets of $\mathrm{AD}$ and save them in TXT format.

\subsection{Prediction and Screening of Drug Targets}

Swiss TargetPrediction database (http://www.swisstargetprediction.ch/) was selected to find the top 30 active components of Astragalus membranaceus and Acorus tatarinowi. In addition, the TCMSP database and SwissTargetPrediction database were integrated to collect the targets of active ingredients of Astragalus membranaceus and Acorus tatarinowii. After redundancy was removed, all the collected targets were imported into bioDBnet software (https://biodbnet-abcc.ncifcrf.gov/) for batch standardization, and screen for non-human targets and calibrate related target names. Then, we obtained the target data set of drug active ingredients and saved it in TXT format.

\subsection{Construction and Analysis of Active Ingredient-Target-Disease Network Diagram}

To elucidate the molecular mechanism of drug pair to treat $\mathrm{AD}$, we constructed a visual network of "ingredients-target-disease" interaction based on the collection of drug targets and disease targets. The collected and sorted Astragalus membranaceus and Acorus tatarinowii ingredients targets were mapped against each other with AD targets to screen out the common composition-disease targets. The screened targets were uploaded to Cytoscape 3.7.2 software (http://www.cytoscape.org/), and then the 
visualization function was used to construct the "Astragalus membranaceus and Acorus tatarinowii "active ingredients-target- disease" network map and save it.

\subsection{GO and KEGG Analysis and the Construction of Target-Pathway Network Diagram}

In this study, we analyzed GO enrichment analysis and KEGG pathway enrichment analysis of drug pair and disease target of AD by using the clusterProfiler enrichment function in $\mathrm{R}$ language, and drew bubble charts and bar charts. On color represented on type of enrichment results, their sizes were positively correlated with the significance. Then we used Cytoscape 3.7.2 visualization software to visualize the signal pathways and the target to construct a "target-pathway" network.

\subsection{Construction of Protein Interaction Network and Network Topol2.6ogy Analysis}

The protein-protein interaction (PPI) network has become an important method to find direct and indirect interactions between targets and targets. Therefore, this study aimed to screen the core targets of Astragalus membranaceus and Acorus tatarinowii in the treatment of $\mathrm{AD}$, using Cytoscape 3.7.2 software. The potential target information was imported into Cytoscape 3.7.2 software, and the high-confidence PPI network information of the active ingredients of Astragalus membranaceus and Acorus tatarinowii and $\mathrm{AD}$ intersection targets were obtained based on the Bisogenet function package. Meanwhile, based on this high-confidence PPI network, Network topology Analysis was performed using the CytoNCA toolkit network analysis to filter core targets based on relevant topology parameters.

\section{Results}

\subsection{Screening of Active Ingredients of Drug Pair}

In this study, 14 active ingredients of Astragalus membranaceus and 2 active ingredients of Acorus calamus were acquired through TCMSP database and literature search. Detailly, the chemical information of each ingredient was tabled according to the number, name, $\mathrm{OB}$ value, $\mathrm{BBB}$ value, and $\mathrm{DL}$ value, and the results were used to construct the dataset of active ingredient information of Astragalus membranaceus and Acorus tatarinowii. Subsequently, we screened the highly active pharmaceutical ingredients according to the criteria of $\mathrm{OB} \geq 30 \%, \mathrm{BBB} \geq 0.3$ and $\mathrm{DL} \geq 0.18$, and obtained 14 active ingredients of Astragalus membranaceus and 2 active ingredients of Acorus tatarinowii, add up to 6 nonrepetitive effectively active ingredients with good ADME properties (table 1).

\subsection{Identification of Alzheimer's Disease Targets}

All target genes associate with AD were searched and converged by the GeneCards and DisGeNET database with "Alzheimer's Disease" as the keyword. We processed and integrated the collected disease targets, deleted redundant and duplicate targets, collected 3397 AD targets, sorted and tabulated them and saved them in TXT format. 
Table 1. Basic information of Astragalus membranaceus and Acorus tatarinowii effectively active ingredients.

\begin{tabular}{|c|c|c|c|c|}
\hline $\begin{array}{l}\text { Molecular } \\
\text { number }\end{array}$ & Effectively active ingredients name & OB & BBB & DL \\
\hline MOL000211 & Mairin & 55.38 & 0.22 & 0.78 \\
\hline MOL000239 & Jaranol & 50.83 & -0.22 & 0.29 \\
\hline MOL000296 & Hederagenin & 36.91 & 0.96 & 0.75 \\
\hline MOL000033 & $\begin{array}{l}\text { (3S,8S,9S,10R,13R,14S,17R)-10,13-dimethyl-17- } \\
{[(2 \mathrm{R}, 5 \mathrm{~S})-5 \text {-propan-2-yloctan-2-yl]- }} \\
2,3,4,7,8,9,11,12,14,15,16,17 \text {-dodecahydro-1H- } \\
\text { cyclopenta[a]phenanthren-3-ol }\end{array}$ & 36.23 & 1.09 & 0.78 \\
\hline MOL000371 & 3,9-di-O-methylnissolin & 53.74 & 0.63 & 0.48 \\
\hline MOL000378 & 7-O-methylisomucronulatol & 74.69 & 0.84 & 0.3 \\
\hline MOL000380 & $\begin{array}{l}\text { (6aR,11aR)-9,10-dimethoxy-6a,11a-dihydro-6H- } \\
\text { benzofurano[3,2-c]chrOmen-3-ol }\end{array}$ & 64.26 & 0.55 & 0.42 \\
\hline MOL000387 & Bifendate & 31.1 & -0.06 & 0.67 \\
\hline MOL000392 & Formononetin & 69.67 & 0.02 & 0.21 \\
\hline MOL000398 & Isoflavanone & 109.99 & 0.17 & 0.3 \\
\hline MOL000438 & $\begin{array}{l}\text { (3R)-3-(2-hydroxy-3,4-dimethoxyphenyl)chroman- } \\
\text { 7-ol }\end{array}$ & 67.67 & 0.34 & 0.26 \\
\hline MOL000442 & 1,7-Dihydroxy-3,9-dimethoxy pterocarpene & 39.05 & -0.04 & 0.48 \\
\hline MOL003576 & $\begin{array}{l}\text { (1R,3aS,4R,6aS)-1,4-bis(3,4-dimethoxyphenyl)- } \\
1,3,3 \mathrm{a}, 4,6,6 \mathrm{a}-\mathrm{H} \text { - } x \text { - }\end{array}$ & 52.35 & 0.83 & 0.62 \\
\hline MOL003578 & Cycloartenol & 38.69 & 1.53 & 0.78 \\
\hline MOL003576 & $\begin{array}{l}\text { (1R,3aS,4R,6aS)-1,4-bis(3,4-dimethoxyphenyl)- } \\
1,3,3 \mathrm{a}, 4,6,6 \mathrm{a}-\text {-HexahydRofuro[4,3-c]furan }\end{array}$ & 52.35 & 0.83 & 0.62 \\
\hline MOL003578 & Cycloartenol & 38.69 & 1.53 & 0.78 \\
\hline
\end{tabular}

\subsection{Screening and Identification of Drug Targets}

A total of 229 Astragalus membranaceus and 69 Acorus tatarinowii drug targets were found through the of TCMSP and SwissTargetPrediction database. After standardization of bioDBnet software and deredundancy, finally, a total of 171 Astragalus membranaceu and Acorus tatarinowii drug targets were collected by standardization of bioDBnet software and deredundancy.

\subsection{Construction of "Active Ingredients-Target-Disease” Network Map and Analysis of Results}

The Astragalus membranaceu and Acorus tatarinowii drug targets were mapped against the collected $\mathrm{AD}$ targets to acquire the targets of the drugs active ingredients of $\mathrm{AD}$ treatment. The drug and disease targets were uploaded to Venny to draw a Venn diagram of the common action targets of drugs and diseases. Finally, we acquired 107 common active targets (figure 1).

The "active ingredient-target-Alzheimer's disease" network was established by Cytoscape3.7.2. Figure 2 represents the 16 active ingredients in Astragalus membranaceu and Acorus tatarinowii that interact with 107 potential targets and thus affect the development of $\mathrm{AD}$, of which only 9 active ingredients can interact with potential targets, they are Mairin;Hederagenin; Isoflavanone;Cycloartenol;(3R)-3-(2hydroxy-3,4-dimethoxyphenyl)chroman-7-ol;(1R,3aS,4R,6aS)-1,4-bis(3,4dimethoxyphenyl)-1,3,3a,4,6,6a-Hexahydrofuro[4,3-c]furan;(6aR,11aR)-9,10- 
dimethoxy-6a,11a-dihydro-6H-benzofurano[3,2-c]chrOmen-3-ol;7-Omethylisomucronulatol;(3S,8S,9S,10R,13R,14S,17R)-10,13-dimethyl-17-[(2R,5S)-5propan-2-yloctan-2-yl]-2,3,4,7,8,9,11,12,14,15,16,17-dodecahydro-1Hcyclopenta[a]phenanthren-3-ol. The results showed that Astragalus membranaceusAcorus tatarinowii drug pair play an effect on the cure of AD by multiple targets, multiple chemical ingredients, and comprehensive regulation.

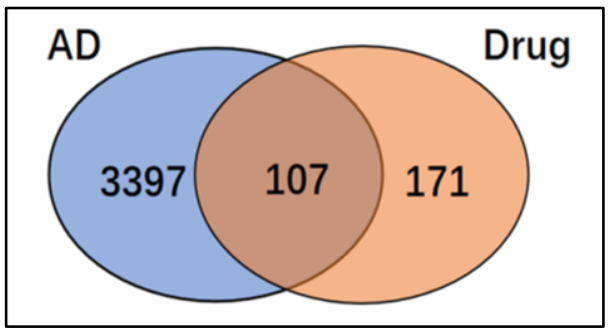

Figure 1. The Venn diagram of the common target of drugs and diseases. Blue is the disease target of Alzheimer's disease; orange is the drug target of Astragalus membranaceus and Acorus tatarinowii; the intersecting part is the common target.

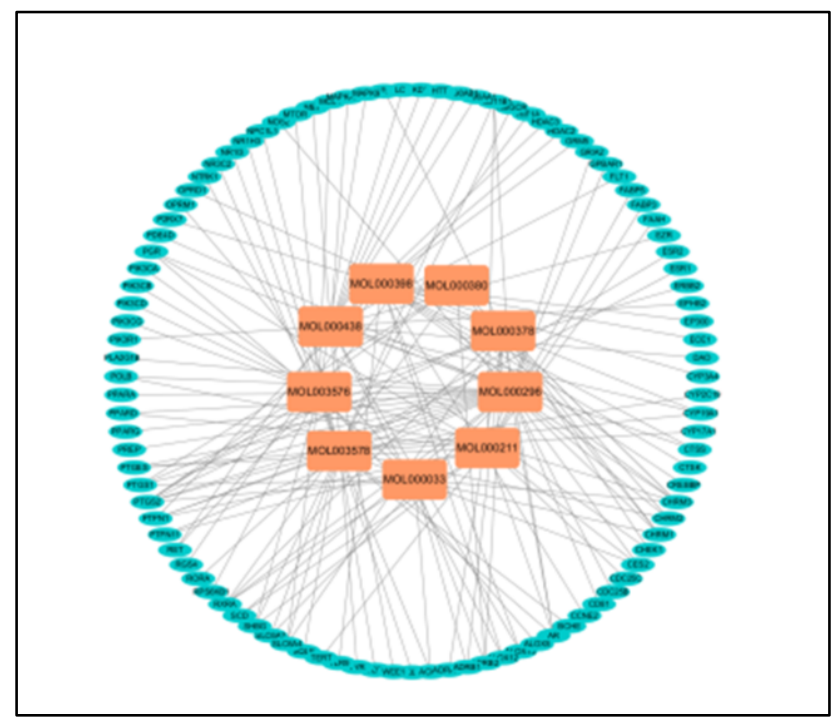

Figure 2. Ingredients-target-disease interaction network diagram. Orange rectangles part is the relevant active ingredient; green ellipse part is the relevant target.

\subsection{GO Function Enrichment Analysis Results}

We used the GO enrichment analysis function in R language to research the 107 target protein-related genes functions involved in the above-mentioned "ingredients-targetdisease" network diagram, then the $60 \mathrm{GO}$ items were selected in the GO enrichment analysis and drawn corresponding bar charts and bubble charts. The results are shown in figure 3 and table 2. As can be seen from table 2, the table involves three parts: biological process (BP), cell composition (CC) and molecular function (MF), and each part selects 10 main go items. 


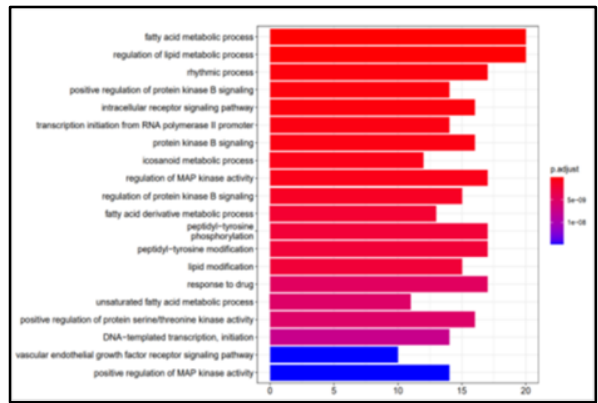

(a)

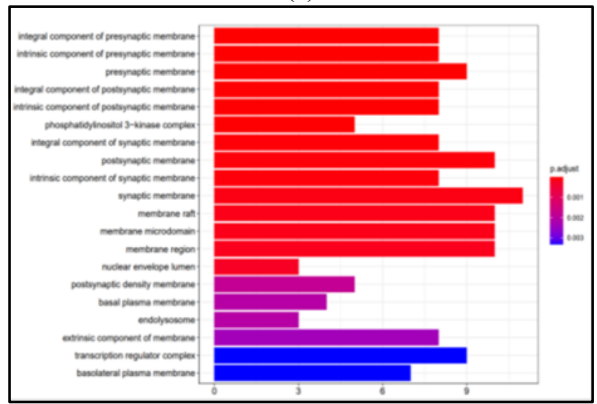

(c)

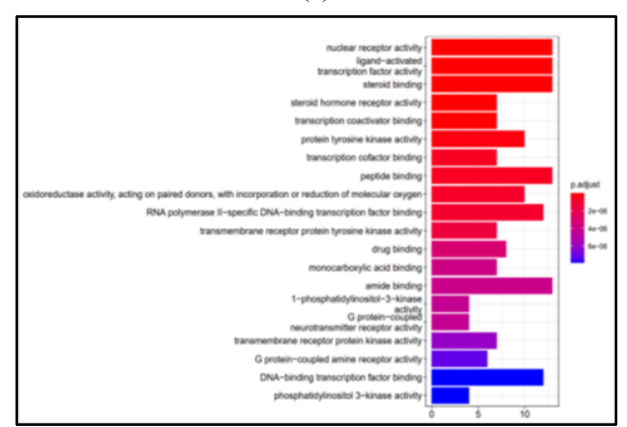

(e)

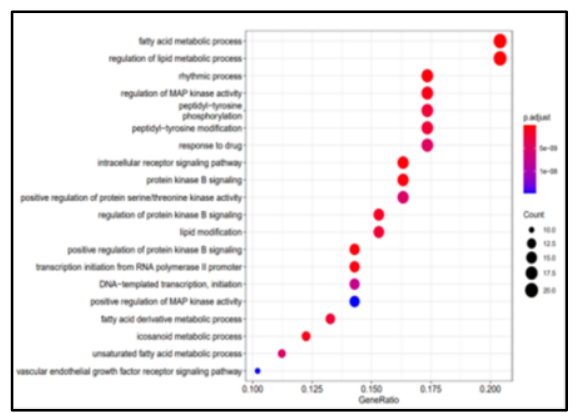

(b)

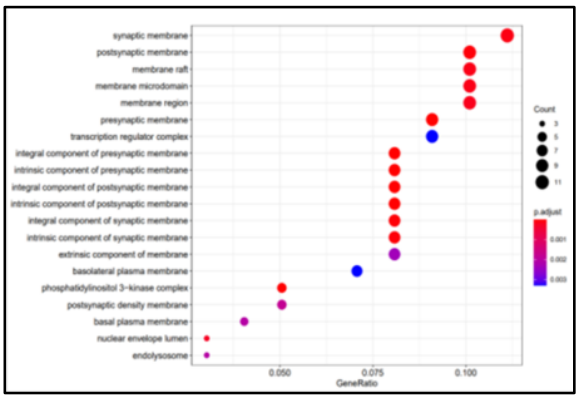

(d)

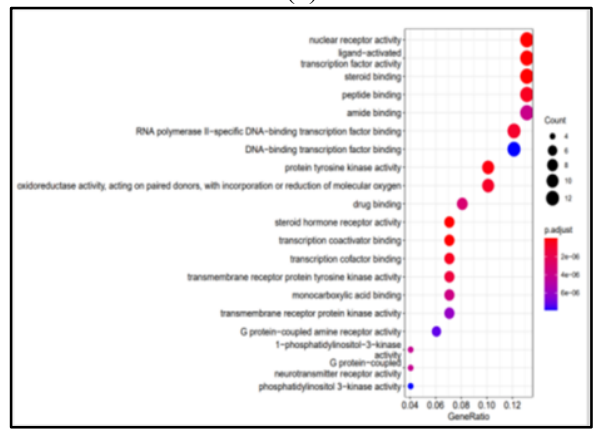

(f)

Figure 3. Histograms and bubble charts of GO enrichment analysis of potential targets of Astragalus membranaceus and Acorus tatarinowii drug pair related to AD. Note, (a) and (b) are bar and bubble chart of BP respectively. (c) and (d) are CC bar and bubble chart respectively. (e) and (f) are MF bar and bubble chart respectively.

\subsection{KEGG Functional Enrichment Analysis Results and the Construction of Target- Pathway Network Diagram}

To illustrate the role of active components of Astragalus membranaceus-Acorus tatarinowii drug pair to treat of AD-related targets in the signaling pathway, the 107 potential targets in the above network diagram through the KEGG enrichment analysis function in R language. The 20 KEGG items were selected in the KEGG enrichment analysis and drawn corresponding bar charts and bubble charts. The 20 signaling pathways obtained are involved in prostate cancer, central carbon metabolism of cancer, thyroid hormone signaling pathway, cAMP signaling pathway, and so on (figure 4). These results indicate that the active ingredients of Astragalus membranaceus and Acorus tatarinowii can affect the development of AD disease through signal pathways such as the thyroid signaling pathway. Further, 107 potential target genes and the involved KEGG pathways were introduced into the software of Cytoscape3.7.2 to construct a "targetpathway" network map, as shown in figure 5. 
Table 2. Major GO entries of potential targets of Astragalus membranaceus and Acorus tatarinowii drug pair related to $\mathrm{AD}$.

\begin{tabular}{|c|c|c|c|c|}
\hline $\begin{array}{l}\text { Term } \\
\text { identification } \\
\text { number }\end{array}$ & Function & \multicolumn{3}{|c|}{ Pvalue Qvalue Count } \\
\hline GOTERM_BP_DIRECT GO:0006631 & Fatty acid metabolism process & $\begin{array}{l}1.31 \mathrm{E}- \\
14\end{array}$ & $\begin{array}{l}2.41 \mathrm{E}- \\
11\end{array}$ & 20 \\
\hline GOTERM_BP_DIRECT GO:0019216 & Regulation of lipid metabolism process & $\begin{array}{l}6.44 \mathrm{E}- \\
14\end{array}$ & $\begin{array}{l}5.94 \mathrm{E}- \\
11\end{array}$ & 20 \\
\hline GOTERM_BP_DIRECT GO:0048511 & Rhythmic process & $\begin{array}{l}3.17 \mathrm{E}- \\
13\end{array}$ & $\begin{array}{l}1.82 \mathrm{E}- \\
10\end{array}$ & 17 \\
\hline GOTERM_BP_DIRECT GO:0051897 & Positive regulation of protein kinase B signaling & $\begin{array}{l}4.33 \mathrm{E}- \\
13\end{array}$ & $\begin{array}{l}1.82 \mathrm{E}- \\
10\end{array}$ & 14 \\
\hline GOTERM_BP_DIRECT GO:0030522 & Intracellular receptor signaling pathway & $\begin{array}{l}4.95 \mathrm{E}- \\
13\end{array}$ & $\begin{array}{l}1.82 \mathrm{E}- \\
10\end{array}$ & 16 \\
\hline GOTERM_BP_DIRECT GO:0006367 & $\begin{array}{l}\text { Transcription initiation of RNA polymerase II } \\
\text { promoter }\end{array}$ & $\begin{array}{l}9.88 \mathrm{E}- \\
13\end{array}$ & $\begin{array}{l}2.71 \mathrm{E}- \\
10\end{array}$ & 14 \\
\hline GOTERM_BP_DIRECT GO:0043491 & Protein kinase B signaling & $\begin{array}{l}1.03 \mathrm{E}- \\
12\end{array}$ & $\begin{array}{l}2.71 \mathrm{E}- \\
10\end{array}$ & 16 \\
\hline GOTERM_BP_DIRECT GO:0006690 & Iconic metabolic process & $\begin{array}{l}1.57 \mathrm{E}- \\
12\end{array}$ & $\begin{array}{l}3.61 \mathrm{E}- \\
10\end{array}$ & 12 \\
\hline GOTERM_BP_DIRECT GO:0043405 & Regulation of MAP kinase activity & $\begin{array}{l}2.00 \mathrm{E}- \\
12\end{array}$ & $\begin{array}{l}4.10 \mathrm{E}- \\
10\end{array}$ & 17 \\
\hline GOTERM_BP_DIRECT GO:0051896 & Regulation of protein kinase B signal transduction & $\begin{array}{l}3.65 \mathrm{E}- \\
12\end{array}$ & $\begin{array}{l}6.73 \mathrm{E}- \\
10\end{array}$ & 15 \\
\hline GOTERM_CC_DIRECT GO:0099056 & The composition of the presynaptic membrane & $\begin{array}{l}3.70 \mathrm{E}- \\
09\end{array}$ & $\begin{array}{l}6.82 \mathrm{E}- \\
07\end{array}$ & 8 \\
\hline GOTERM_CC_DIRECTGO:0098889 & Intrinsic ingredients of presynaptic membrane & $\begin{array}{l}9.33 \mathrm{E}- \\
09\end{array}$ & $\begin{array}{l}8.59 \mathrm{E}- \\
07\end{array}$ & 8 \\
\hline GOTERM_CC_DIRECTGO:0042734 & Presynaptic membrane & $\begin{array}{l}9.73 \mathrm{E}- \\
08\end{array}$ & $\begin{array}{l}5.97 \mathrm{E}- \\
06\end{array}$ & 9 \\
\hline GOTERM_CC_DIRECTGO:0099055 & The composition of the postsynaptic membrane & $\begin{array}{l}1.40 \mathrm{E}- \\
07\end{array}$ & $\begin{array}{l}6.45 \mathrm{E}- \\
06\end{array}$ & 8 \\
\hline GOTERM_CC_DIRECTGO:0098936 & Intrinsic ingredients of the postsynaptic membrane & $\begin{array}{l}1.94 \mathrm{E}- \\
07\end{array}$ & $\begin{array}{l}7.14 \mathrm{E}- \\
06\end{array}$ & 8 \\
\hline GOTERM_CC_DIRECT GO:0005942 & Phosphatidylinositol 3-kinase complex & $\begin{array}{l}3.24 \mathrm{E}- \\
07\end{array}$ & $\begin{array}{l}9.93 \mathrm{E}- \\
06\end{array}$ & 5 \\
\hline GOTERM_CC_DIRECTGO:0099699 & Ingredients of the synaptic membrane & $\begin{array}{l}1.15 \mathrm{E}- \\
06\end{array}$ & $\begin{array}{l}3.03 \mathrm{E}- \\
05\end{array}$ & 8 \\
\hline GOTERM_CC_DIRECTGO:0045211 & Postsynaptic membrane & $\begin{array}{l}1.56 \mathrm{E}- \\
06\end{array}$ & $\begin{array}{l}3.60 \mathrm{E}- \\
05\end{array}$ & 10 \\
\hline GOTERM_CC_DIRECTGO:0099240 & Synaptic membrane ingredients & $\begin{array}{l}2.02 \mathrm{E}- \\
06\end{array}$ & $\begin{array}{l}4.14 \mathrm{E}- \\
05\end{array}$ & 8 \\
\hline GOTERM_CC_DIRECTGO:0097060 & Synaptic membrane & $\begin{array}{l}4.19 \mathrm{E}- \\
06\end{array}$ & $\begin{array}{l}7.72 \mathrm{E}- \\
05\end{array}$ & 11 \\
\hline GOTERM_MF_DIRECTGO:0004879 & Nuclear receptor activity & $\begin{array}{l}7.74 \mathrm{E}- \\
19\end{array}$ & $\begin{array}{l}1.02 \mathrm{E}- \\
16\end{array}$ & 13 \\
\hline GOTERM_MF_DIRECTGO:0098531 & Ligand activates transcription factor activity & $\begin{array}{l}7.74 \mathrm{E}- \\
19\end{array}$ & $\begin{array}{l}1.02 \mathrm{E}- \\
16\end{array}$ & 13 \\
\hline GOTERM_MF_DIRECTGO:0005496 & Steroid binding & $\begin{array}{l}1.53 \mathrm{E}- \\
14\end{array}$ & $\begin{array}{l}1.34 \mathrm{E}- \\
12\end{array}$ & 13 \\
\hline GOTERM_MF_DIRECTGO:0003707 & Steroid Hormone Receptor & $\begin{array}{l}6.48 \mathrm{E}- \\
11\end{array}$ & $\begin{array}{l}4.27 \mathrm{E}- \\
09\end{array}$ & 7 \\
\hline GOTERM_MF_DIRECTGO:0001223 & Transcriptional coactivator binding & $\begin{array}{l}1.52 \mathrm{E}- \\
10\end{array}$ & $\begin{array}{l}7.99 \mathrm{E}- \\
09\end{array}$ & 7 \\
\hline GOTERM_MF_DIRECTGO:0004713 & Protein tyrosine kinase activity & $\begin{array}{l}2.96 \mathrm{E}- \\
09\end{array}$ & $\begin{array}{l}1.30 \mathrm{E}- \\
07\end{array}$ & 10 \\
\hline GOTERM_MF_DIRECTGO:0001221 & Transcription cofactor binding & $\begin{array}{l}1.02 \mathrm{E}- \\
08\end{array}$ & $\begin{array}{l}3.64 \mathrm{E}- \\
07\end{array}$ & 7 \\
\hline GOTERM_MF_DIRECTGO:0042277 & Peptide binding & $\begin{array}{l}1.11 \mathrm{E}- \\
08\end{array}$ & $\begin{array}{l}3.64 \mathrm{E}- \\
07\end{array}$ & 13 \\
\hline GOTERM_MF_DIRECTGO:0016705 & $\begin{array}{l}\text { Oxidoreductase activity, acting on the } \\
\text { incorporation or reduction of molecular oxygen in } \\
\text { pairs of donors }\end{array}$ & $\begin{array}{l}1.72 \mathrm{E}- \\
08\end{array}$ & $\begin{array}{l}5.04 \mathrm{E}- \\
07\end{array}$ & 10 \\
\hline GOTERM_MF_DIRECTGO:0061629 & $\begin{array}{l}\text { RNA polymerase II specific DNA binding } \\
\text { transcription factor binding }\end{array}$ & $\begin{array}{l}2.11 \mathrm{E}- \\
08\end{array}$ & $\begin{array}{l}5.56 \mathrm{E}- \\
07\end{array}$ & 12 \\
\hline
\end{tabular}




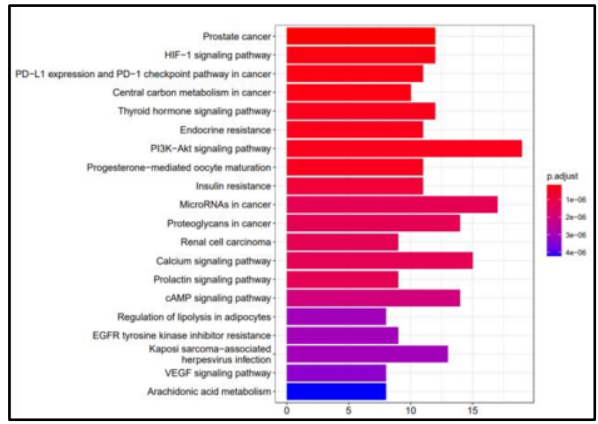

(a)

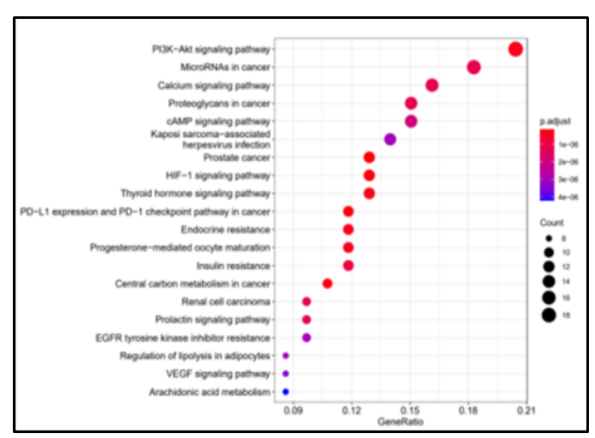

(b)

Figure 4. bar chart and bubble chart of KEGG enrichment analysis of drug pair.

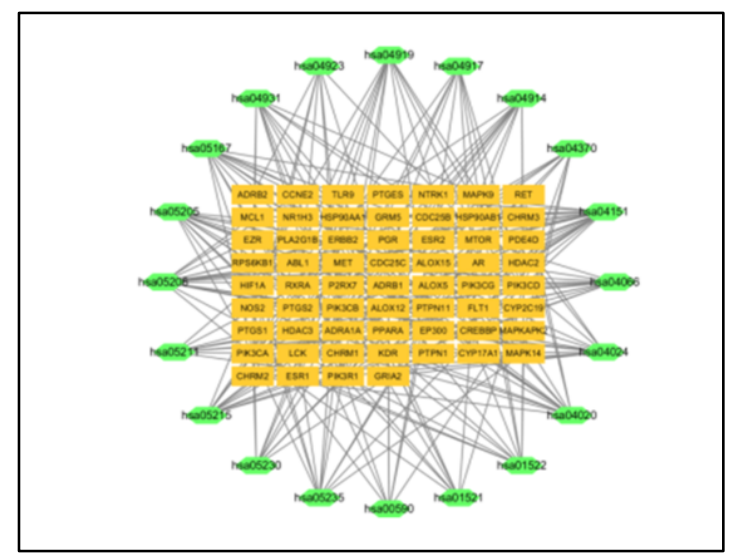

Figure 5. "Target-pathway" network diagram. Yellow rectangles part is the target enriched to the signal pathway; green ellipse part is the signal pathway.

\subsection{Results of Protein Interaction Network and Network Topology Analysis}

In order to screen the core targets of Astragalus membranaceus and Acorus tatarinowii for the treatment of $\mathrm{AD}, 107$ potential targets were imported into Cytoscape 3.7.2software (http://www.cytoscape.org/). Next, high confidence PPI network information of active ingredients Astragalus membranaceus and Acorus tatarinowii and disease intersection targets were obtained by Bisogenet function package (figure 6). Based on this PPI network in Cytoscape, the network was topological analyzed though the CytoNCA toolkit, and the corresponding topological parameters $\mathrm{DC}, \mathrm{BC}, \mathrm{NC}$ were $61,458,34$. The core targets were screened based on the degree centrality (DC) value before betweenness centrality (BC) and drawn a network diagram, and the network graph was drawn (figure 7). Figure 7 is the network topology analysis diagram. The results showed that the red nodes ESR1 (estrogen receptor 1), HSP90AB1 (heat shock protein 90 alpha family class B member 1), NTRK1 (neurotrophic tyrosine kinase receptor 1) and HSP90AA1 (heat shock protein 90 alpha family class A member 1), and are core target proteins, and the yellow nodes are related genes of the core target proteins. Moreover, topological parameters of HSP90AA1, HSP90AB1, NTRK1 and 
ESR1 meet the above card values, so they can be used as the core targets of Astragalus membranaceus and Acorus tatarinowii in the treatment of AD.

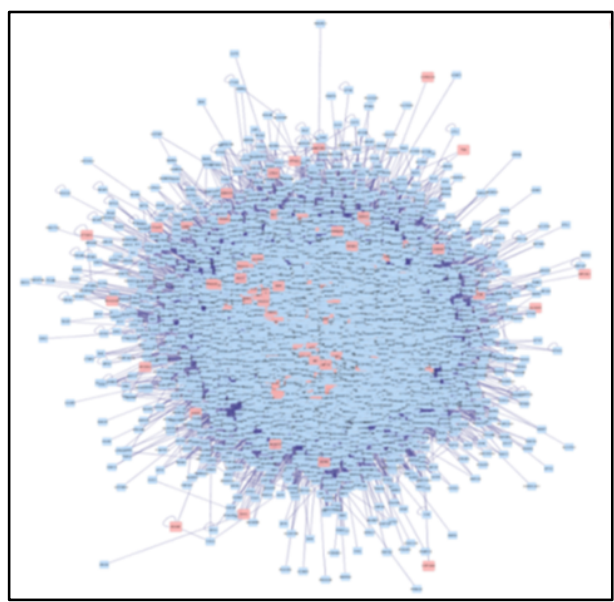

Figure 6. High-confidence interaction network diagram (PPI) of target proteins of Astragalus membranaceus and Acorus tatarinowii in the treatment of AD.

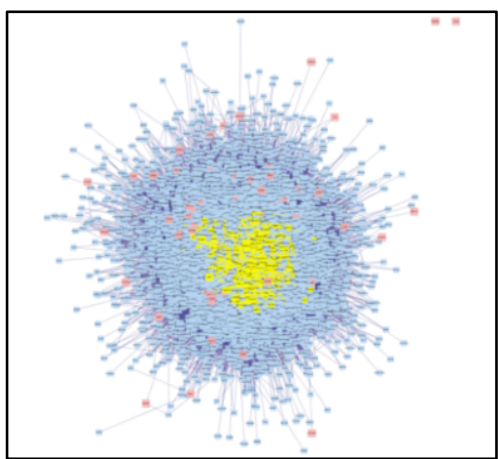

(a)

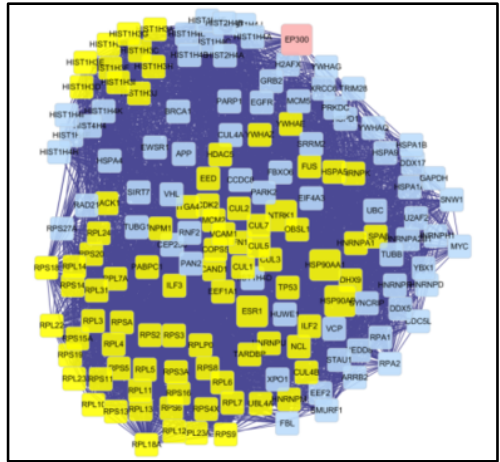

(c)

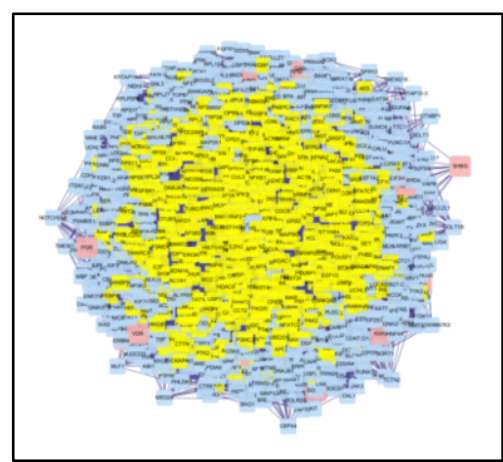

(b)

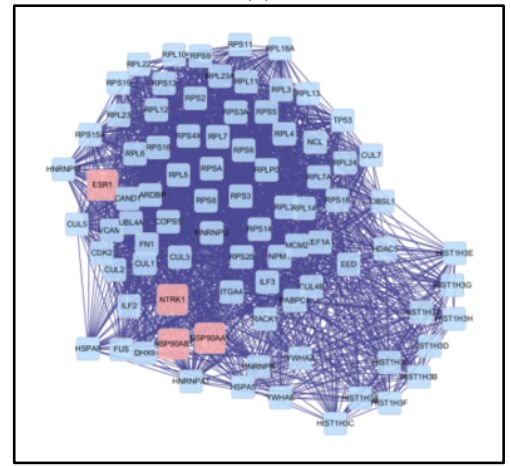

(d)

Figure 7. Core target map of Astragalus membranaceus and Acorus tatarinowii in the treatment of AD in topological analysis. Note, Red rectangles part is the input target gene; yellow rectangles part is the input target related protein gene A; Based on PPI network and according to the screening criteria of DC > 61; B: (a) creates a subnetwork and draws it according to the screening criteria of $\mathrm{BC}>458$; $\mathrm{C}$ : (b) creates a subnetwork and draw it according to the standard of NC>34; D: (c) creates a subnetwork. 


\section{Discussion}

$\mathrm{AD}$ is a neurodegenerative disease with progressive cognitive decline, which has become the third major killer to human life and health after tumor and cardiovascular disease $[21,22]$. AD seriously affects the mental and physical health of the elderly. Therefore, it is important that studying the pathogenesis of $\mathrm{AD}$ to its prevention and treatment. In modern medicine, western medicine therapy can only delay and improve the occurrence of symptoms, but can't completely cure the disease. As an auxiliary therapy to treat AD, TCM has the advantage of anti-AD additives, and the welltolerated ingredients are safer in $\mathrm{AD}$ patients. None of these properties exist in currently approved drugs [23]. Therefore, it is of great value to analyze the medication law of TCM therapy AD.

In this study, the TCMSP and SwissTragetPrediction database were used to screen all the effective active components and related targets related to Astragalus membranaceus and Acorus tatarinowii drug pair, and then the targets were standardized by biodbnet and other softwares. We collected the target genes of $\mathrm{AD}$ through DisGeNET database, and mapped to the drug and disease targets, and drawn the "ingrediens-target-disease" network diagram, and constructed the PPI protein interaction network, CytoNCA network topology analysis, GO function enrichment analysis, KEGG signal pathway enrichment analysis, and KEGG relationship network diagram.

The "active ingredients-targets-disease" network diagram (figure 2) showed that Astragalus membranaceus and Acorus tatarinowii could treat AD by acting on 107 targets, including NTRK1, ESR1, AKR1B10, PTPN1, HSD11B1, PTGES, NR1H4 and so on. The screened potential drug targets may be one of the causes of AD. To further explain the role of gene functions and signaling pathways involved in the related targets of Astragalus membranaceus and Acorus tatarinowii in the treatment of AD, the potential targets of drug pair to treat of $\mathrm{AD}$ were analyzed by $\mathrm{GO}$ analysis and KEGG analysis. GO analysis results (figure 3) and KEGG analysis results (figure 4) revealed that relevant drug targets mainly concentrated in the central carbon metabolism, vascular endothelial growth factor and fatty acid metabolism signaling pathways of cancer. And a large number of experiments have revealed that the emerge of AD is strongly linked to cancer diseases and vascular factors. The above results showed that Astragalus membranaceus and Acorus tatarinowii treated AD comprehensively through multiple ways and multiple pathways. The results also conform to the features of "multi-ingredients and multi-target and multi-pathway" in TCM therapy, and afford a theoretical reference for the subsequent study of $\mathrm{AD}$.

In this study, in order to clarify the core targets of Astragalus membranaceusAcorus tatarinowii drug pair in the treatment of $\mathrm{AD}$, we built a high confidence PPI network of Astragalus membranaceus and Acorus tatarinowii to treat $\mathrm{AD}$ by the method of network pharmacology (figure 6), and analyzed the network topology (figure7). The results showed that ESR1, NTRK1, HSP90AA1 and HSP90AB1 were the core targets of Astragalus membranaceus and Acorus tatarinowi in the treatment of AD. These targets are possible etiologic factors in the pathogenesis of AD.

In addition, this study still has certain limitations. For example, when we used bioinformatics tools to retrieve the root of Astragalus membranaceus and Acorus tatarinowii and $\mathrm{AD}$ targets, the introduction of a large amount of data still forecast target related active ingredients. Therefore, the follow-up research Astragalus membranaceus and Acorus tatarinowii to treat $\mathrm{AD}$ the key targets of therapy and 
related pathways also only to predict, and it is still short of follow-up molecular dynamics study and animal experimental support. Based on the method of network pharmacology, this study explores how traditional Chinese medicine can treat Astragalus membranaceus and Acorus tatarinowi with the features of "multi-ingrediens and multi-targets and multi-pathway" to treat $\mathrm{AD}$ systematically. This experiment provides the theoretical basis and research ideas for studying the pathogenesis and drug development of AD.

\section{Conclusion}

In this research, we used bioinformatics tools to search the targets of Astragalus membranaceus, Acorus tatarinowii and AD, and classified and sorted them, and finally predicted the key targets and related pathways of Astragalus membranaceus and tatarinowii in the treatment of $\mathrm{AD}$.

\section{Acknowledgments}

The study was supported by Liaoning provincial key R \& D project (No. 2020JH2/10300114 and 2019JH8/10300057).

\section{References}

[1] Zhang MK and Yu XY. Epidemiology and preventive measures of senile dementia. Shequ Yixue Zazhi (Chinese). [J],2005(11):37-38

[2] Song X, Hong YR, and Hu QY. Research progress in the pathogenesis and mechanism of Alzheimer's disease. Linchuang he Shiyan Yixue Zazhi(Chinese). [J],2015,14(10):871-87

[3] Yang XM, Bao TH, and Ruan Z. An overview of risk factors for Alzheimer's disease. Sichuang Jingshen Weisheng (Chinese). [J],2020,3306:560-565.

[4] GBD 2016 Neurology Collaborators. Global, regional, and national burden of neurological disorders, 1990-2016: a systematic analysis for the Global Burden of Disease Study 2016.[J] .Lancet Neurol, 2019, 18: 459-480.

[5] Alavi Marcel V.Tau phosphorylation and OPA1 proteolysis are unrelated events: Implications for Alzheimer's Disease.[J] . Biochim Biophys Acta Mol Cell Res, 2021, undefined: 119116.

[6] Lai LM, Ling CX, Zhang XF and Hu CH. Research progress in the pathogenesis of Alzheimer's disease(Chinese).[J].Shangqiu Shifan Xuebao,2018,34(12):27-29.

[7] Ferreira-Vieira TH, Guimaraes IM, Silva FR. Ribeiro FM (2016) Alzheimer's disease: targeting the cholinergic system. Curr Neuropharmacol 14:101-115.

[8] Arnsten Amy F T, Datta Dibyadeep, Tredici Kelly Del et al. Hypothesis: Tau pathology is an initiating factor in sporadic Alzheimer's disease.[J] . Alzheimers Dement, 2021, 17: 115-124.

[9] Li XY and Zang M. Effect of Astragalus Polysaccharide on Alzheimer's disease model rats and its mechanism [J]. Shijie Zuixin Yixue Xinxi Wenzhai(Chinese),2019,1944:27-28

[10] Birks Jacqueline S, Harvey Richard J. Donepezil for dementia due to Alzheimer's disease.[J] .Cochrane Database Syst Rev, 2018, 6: CD001190.

[11] Wang XY, Sun GQ, Feng T et al. Sodium oligomannate therapeutically remodels gut microbiota and suppresses gut bacterial amino acids-shaped neuroinflammation to inhibit Alzheimer's disease progression.[J] .Cell Res, 2019, 29: 787-803.

[12] Kook S-Y et al. High-dose of vitamin C supplementation reduces amyloid plaque burden and ameliorates pathological changes in the brain of 5XFAD mice.[J]. Cell death \& disease, 2014, $5: \mathrm{e} 1083$.

[13] Takahashi Ito Kaori et al. Memantine inhibits $\beta$-amyloid aggregation and disassembles preformed $\beta$ amyloid aggregates [J]. Biochemical and Biophysical Research Communications, 2017, 493(1) : 158163. 
[14] Ossenkoppele Rik et al.Tau PET patterns mirror clinical and neuroanatomical variability in Alzheimer's disease.[J]. Brain: a journal of neurology, 2016, 139(Pt 5) : 1551-67.

[15] Yang CC, Jia XY, Zhang L, Li YL, Zhang ZJ, Li L, Zhang . Shenqi Xingnao Granules ameliorates cognitive impairments and Alzheimer's disease-like pathologies in APP/PS1 mouse model[J].Chinese Herbal Medicines, 2020,12(04):421-429.

[16] Chong CM, Su H, Lu JJ et al. Salvia miltiorrhiza The effects of bioactive ingredientss from the rhizome of (Danshen) on the characteristics of Alzheimer's disease.[J] .Chin Med, 2019, 14: 19.

[17] Ma GX, Ding QP and Deng HH et al. Study on the Efficacy and Mechanism of Astragalus Polysaccharide on Alzheimer's Disease Model Rats [J].Cuzhong yu Shenjing Jibing(Chinese),2017,24(4):323-327.

[18] Tian SM, Ma YX and Sun LZ et al. Different medicinal parts of Acorus tatarinowii improves the cognitive function of Alzheimer's disease model mice.[J].Zhongguo Bingli Shengli Zazhi(Chinese),2012,28(1):159-162,167.

[19] Song KL, Zhang QQ, Wang XH and Wang YX. Research on the medication law of modern traditional Chinese medicine in the treatment of Alzheimer's disease based on Data Mining [J]. Shijie Kexue Jishu-Zhongyiyao Xiandaihua(Chinese),2019,21(07):1424-14,29.

[20] Mao LS and Zhu XH. Application progress of network pharmacology in the field of traditional Chinese medicine[J]. Zhongyiyao Guanli Zazhi(Chinese),2021,29(13):98-102.

[21] Harsh Sancheti et al. Age-dependent modulation of synaptic plasticity and insulin mimetic effect of lipoic acid on a mouse model of Alzheimer's disease.[J]. PLoS ONE, 2017, 8(7)

[22] Chen ZC and Zhong CJ. Decoding Alzheimer's disease from perturbed cerebral glucose metabolism: implications for diagnostic and therapeutic strategies.[J]. Progress in neurobiology, 2013, 108:21-43.

[23] Yang, W. T., Zheng, X. W., Chen, S., Shan, C. S., Xu, Q. Q., Zhu, J. Z., et al. Chinese herbal medicine for Alzheimer's disease: Clinical evidence and possible mechanism of neurogenesis. Biochem. Pharmacol. 141, 143-155. 